\title{
La Révolution française
}

Cahiers de l'Institut d'histoire de la Révolution française

$22 \mid 2022$

L'étranger en révolution(s)

\section{Un retour malheureux : l'Italien Giambattista Rotondo, une « machine ouvrière » dans l'Europe en révolution}

Paolo Conte

\section{(2) OpenEdition}

Journals

Édition électronique

URL : https://journals.openedition.org//rf/6162

DOI : $10.4000 /$ Irf.6162

ISSN : 2105-2557

Éditeur

IHMC - Institut d'histoire moderne et contemporaine (UMR 8066)

Référence électronique

Paolo Conte, « Un retour malheureux : I'Italien Giambattista Rotondo, une « machine ouvrière » dans I'Europe en révolution », La Révolution française [En ligne], 22 | 2022, mis en ligne le 20 janvier 2022, consulté le 23 janvier 2022. URL : http://journals.openedition.org//rf/6162 ; DOI : https://doi.org/ $10.4000 /$ Irf.6162

Ce document a été généré automatiquement le 23 janvier 2022.

(c) La Révolution française 


\title{
Un retour malheureux : l'Italien Giambattista Rotondo, une « machine ouvrière » dans l'Europe en révolution
}

\author{
Paolo Conte
}

1 En juillet 1797, quelques semaines après la proclamation de la République cisalpine, plusieurs journaux, à Milan, annoncent la parution d'un étrange volume intitulé Memorie storiche del professore Gio. Bat. Rotondo et dédié au " peuple italien » ainsi qu'aux «patriotes de tout l'univers $»^{1}$. L'auteur, un professeur de langues natif de Monza du nom de Giambattista Rotondo, fort de sa participation à plusieurs révolutions à travers toute l'Europe, y raconte les nombreuses mésaventures de sa vie à partir de 1782, date à laquelle il est contraint de s'enfuir de Milan du fait de ses divergences avec l'administration autrichienne, jusqu'en 1796, lorsque sa détention dans les prisons du Royaume de Sardaigne prend fin grâce à l'intervention de l'armée française. Paru chez l'éditeur Carlo Civati, le livre est salué surtout par les nouveaux journaux patriotiques nés à la faveur de l'entrée des troupes républicaines dans le Milanais : par exemple, le Giornale de' Patrioti d'Italia, décrit l'auteur comme un "vrai martyr et apôtre volontaire de la liberté ", et présente l'ouvrage comme un « guide pour tous ceux qui se trouvent dans un pays en révolution $»^{2}$.

2 Avec cette autobiographie rédigée en plein cœur du Triennio (c'est-à-dire pendant la phase où la péninsule entre en contact avec les armées républicaines), Rotondo envisage de rendre publiques les expériences qu'il a menées à travers toute l'Europe afin d'obtenir des charges dans les nouvelles institutions républicaines. D'ailleurs, bien que natif du Milanais, il n'est pas connu dans les milieux patriotiques italiens, sa carrière s'étant déroulée pour l'essentiel à l'étranger.

3 Le cas de ce professeur et de ces mémoires parues à la suite de sa réapparition en Italie nous amène à poser la question des « étrangers en révolution(s) » dans une perspective particulière, celle du retour. Il s'agit ainsi de réfléchir sur ces étrangers, non seulement 
à l'aune de leur action concrète dans d'autres contextes nationaux, mais aussi par le rôle qu'ils jouent, une fois rentrés dans leurs patries, grâce aux compétences acquises et à la réputation construite au fil de leur participation successive à différents processus révolutionnais. En adoptant une approche essentiellement biographique, nous nous proposons d'enquêter notamment sur les conséquences que cet engagement à l'étranger peut avoir dans la lutte politique du pays d'origine. Considérer ces hommes et ces femmes non seulement comme spectateurs des bouleversements politiques auxquels ils sont confrontés, mais aussi comme des acteurs à part entière du processus révolutionnaire revient moins à interroger les discours portés à leur égard dans le pays d'arrivée que l'activité concrète qu'ils y mènent.

4 Certes, les étrangers en révolution contribuent de façon importante aux événements des pays où ils se trouvent amenés à agir, mais cette participation leur permet aussi d'entamer un processus de formation politique et de construction de rapports humains qui s'avère décisif pour la suite de leur carrière. Ainsi, dans ces circulations révolutionnaires qui dessinent des espaces originaux, ils participent à la construction d'une cité nouvelle, fût-ce loin de leur patrie. Leur séjour à l'étranger peut en fait avoir des répercussions remarquables dans l'élaboration de leurs cultures politiques nationales respectives, tant par les publications qu'ils y font circuler que par les activités qu'ils y mènent à leur retour.

5 Ces répercussions dans le pays d'origine ont d'ailleurs joué un rôle important d'un point de vue historiographique. Elles ont été tantôt soulignées tantôt refusées par les différentes écoles historiographiques, dans des visées bien différentes. Le cas italien est fort emblématique à cet égard. Tout au long $\mathrm{du} \mathrm{XIX}^{\mathrm{e}}$ siècle, et plus encore sous le fascisme, les recherches n'ont que peu examiné la contribution des Italiens à la Révolution française afin de dénier toute filiation du Risorgimento (à savoir le processus amenant à l'unité de la péninsule) avec les conquêtes de 1789 et, ce faisant, pour mieux légitimer, du point de vue culturel autant qu'historique, la dimension autochtone du mouvement national italien. Après la Seconde Guerre mondiale, dans un contexte renouvelé, une nouvelle lecture issue d'une matrice démocratique s'est affirmée en mettant en valeur les liaisons entre patriotisme italien et Révolution française ${ }^{3}$. C'est à cette époque que de nouvelles recherches ont souligné le rôle que le patriote toscan Filippo Buonarroti a joué dans la diffusion en Italie des principes égalitaires, après avoir œuvré en Corse et à Paris. Il en est résulté la thèse d'un jacobinisme italien structuré autour du " robespierrisme de Buonarroti », c'est-à-dire entièrement lié à ce révolutionnaire et, par lui et à rebours, à Robespierre. Cela a permis de présenter l'activité des patriotes italiens au cours du Triennio comme fortement influencée par la culture politique dominante en France sous l'hégémonie robespierriste ${ }^{4}$.

Sans entrer dans les détails de la carrière en France de Buonarroti (qui nous semble davantage liée au Corse Christophe Saliceti qu'à Robespierre et dont le succès historiographique a été favorisé surtout par la parution, en 1828, de son texte célèbre sur la Conspiration des Égaux ${ }^{5}$ ), nous nous bornerons à souligner que la première historiographie démocratique italienne s'est résolument structurée autour de la figure de cet «étranger-ci en révolution » et de ses liaisons avec les élites révolutionnaires françaises. En somme, tant au cours de la Révolution que par la suite, tant dans la lutte politique de l'époque que dans l'historiographie depuis, cette présence étrangère a joué un rôle majeur dans le contact entre la France révolutionnaire et d'autres contextes nationaux ${ }^{6}$. 
7 Si le cas de Buonarroti a été, du moins du côté italien, le plus étudié, d'autres parcours d'exilés patriotes permettent toutefois de relativiser la « représentativité » de son cas. À travers l'analyse d'une autre biographie, celle de Giambattista Rotondo, nous nous proposons de réfléchir aux conséquences que cette présence peut entraîner sur une durée plus longue et dans un espace géographique plus vaste. Il s'agit en même temps de montrer la profondeur sociale et les déclinaisons politiques de cette présence étrangère dans la France en révolution au-delà des élites les plus influentes. Il sera donc opportun d'enquêter sur les modalités par lesquelles, dès son retour, ce professeur utilise son passé pour gagner de nouvelles protections et pour obtenir des charges en Italie. Puis, en analysant sa trajectoire à travers toute l'Europe et en particulier en France, nous saisirons les correspondances, plus ou moins véridiques, entre son récit et l'activité qu'il a véritablement menée à l'étranger. Pour finir, nous tâcherons d'évaluer si et comment, par la suite, ces expériences ont favorisé sa carrière dans la péninsule.

\section{Une réapparition inattendue dans Milan républicanisé}

Dès le printemps 1797, lorsque Rotondo se réfugie à Gênes après sa libération des prisons piémontaises, les milieux révolutionnaires de la ville ligure s'activent tout de suite pour favoriser la réussite de ses objectifs et lui faire obtenir des charges. Par exemple, tandis que le secrétaire de la légation française Joseph Villetard, sous la poussée du commissaire Guillaume-Charles Faipoult, écrit au général Bonaparte pour soutenir la demande de Rotondo visant à obtenir le dédommagement de la part de la cour de Turin pour les années passées en prison ${ }^{7}$, le patriote napolitain Andrea Carizzi le recommande, fin mars, à son concitoyen demeurant à Milan Francesco Saverio Salfi. Ce n'est donc pas un hasard si, quelques mois plus tard, ses Memorie sont si appréciées par un journal qui est dirigé par deux des exilés napolitains les plus en vue : Matteo Galdi et Giuseppe Abamonti ; cela montre en fait la capacité de Rotondo à gagner la confiance des hommes du parti républicain, dans lequel militait la plupart des exilés méridionaux. En outre, la lettre de Carizzi démontre bien l'intensité de l'engagement politique de ce parti républicain dans le contexte d'enthousiasme général qui traverse la péninsule suite à l'entrée des armées françaises :

Le chargé de cette lettre est le citoyen Rotondo. Il est l'une des victimes du despotisme sarde : au bout d'une détention longue et pénible, il a vécu la joie, on ne sait pas comment, de respirer et voir à nouveau cette lumière qu'on lui avait interdite pendant plusieurs années. Il se rend maintenant dans le sol de la liberté. Or, loin des pièges et des griffes de la tyrannie, il veut rendre publique à tout le monde la persécution injuste et horrible qu'il a souffert par le despotisme du plus lâche des tyrans. Il a besoin de ta plume et de ton éloquence virile, non parce que la vérité a besoin des couleurs pour gagner du terrain, mais pour montrer pleinement les horreurs du pouvoir arbitraire. Je te le recommande avec la chaleur qui mérite un ami de la bonne cause, un patriote de la Liberté. [...] Ta plume est pour les despotes plus destructrice et mortelle que le fer actuel des républicains.

Les affaires vont mieux de plus en plus dans notre Italie. À la tempête la plus horrible suit pour une fois un calme rassurant pour le genre humain ! Je l'espère, ou mieux, j'en suis sûr. Vive la liberté. Vive la République italienne. Vive le bonheur et la rédemption de l'Univers ${ }^{8}$.

9 C'est fort de cette double recommandation que Rotondo rejoint Milan quelques semaines plus tard, alors que ses Memorie storiche paraissent sous les presses de 
l'imprimeur français Villetard, l'un des hommes les plus proches du mouvement républicain italien et l'un des animateurs, avec l'éditeur Civati, du Giornale de' Patrioti d'Italia. Ces réseaux s'avèrent ainsi décisifs pour l'écho immédiat que rencontre le texte de Rotondo dans la toute jeune République cisalpine.

En outre, si dans un premier moment ce professeur s'était proposé - comme il l'écrit à son cousin Fedele Sopransi dès son arrivée à Milan - de dédier son travail «à l'Alexandre français, le général en chef Bonaparte ", il change d'avis par la suite et décide de supprimer toute référence au chef de l'Armée d'Italie, quand bien même sa libération n'est due qu'à l'entrée dans la péninsule des troupes menées par le général corse. Cette autocensure est probablement due à la signature, à la mi-avril 1797, du traité de Leoben, lequel, en anticipant de quelques mois la paix de Campoformio, prouve l'inclination désormais attestée de la France à sacrifier ses possessions vénitiennes sur l'autel de la paix avec l'Autriche, suscitant la première crise de confiance avec les patriotes italiens'.

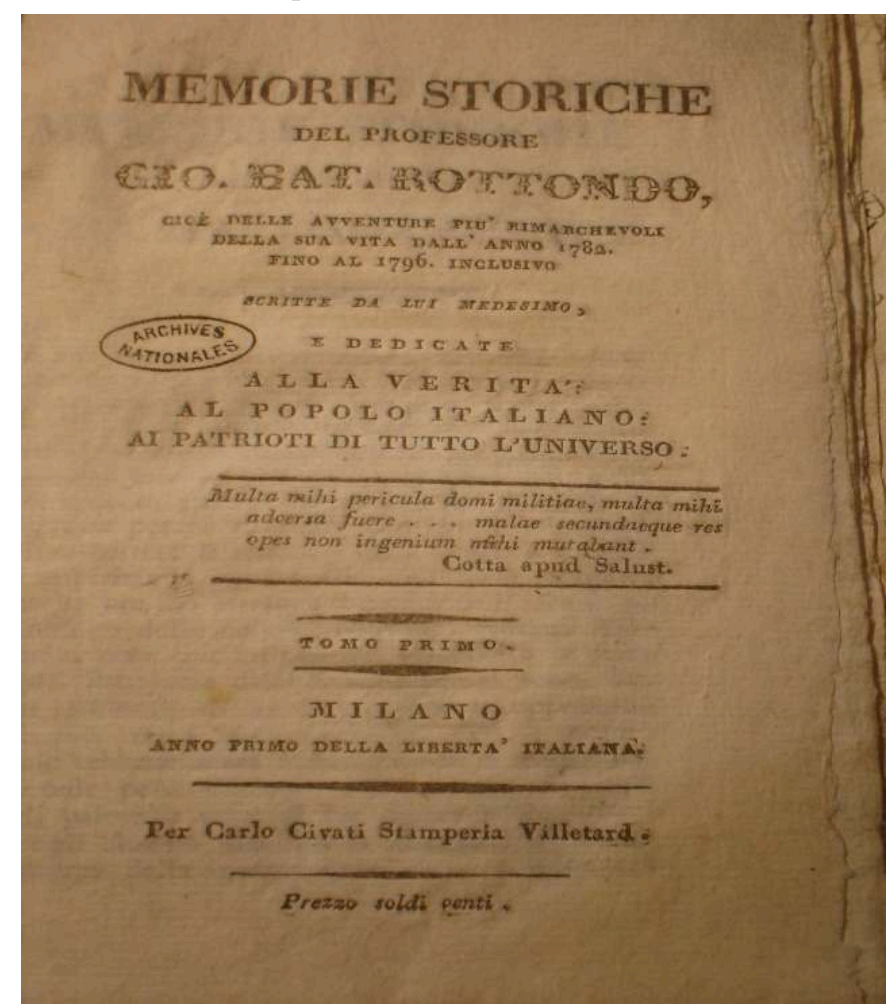

Giambattista Rotondo, Memorie storiche del professore Gio. Bat. Rotondo, cioè delle avventure più rimarchevoli Della sua vita dall'anno 1782 fino al 1796 inclusivo, Milan, Civati, 1797 @ AN, F/7, carton 6155, dossier 1133, pl. 2

11 Initialement rédigée à la première personne et en français pour pouvoir être envoyée comme mémoire personnel à Paris auprès du Directoire, la version définitive de cette autobiographie, imprimée à Milan, est finalement publiée en italien et à la troisième personne ${ }^{10}$. Ainsi, alors qu'elle est commencée dans le but d'obtenir le soutien du gouvernement français à l'égard de la pétition demandant le remboursement des pertes subies par le Roi de Sardaigne, l'écriture de ce texte change d'objectif et de destinataires au fur et à mesure des événements : dans le Milanais républicanisé, ce texte devient un moyen par lequel son auteur se propose de conquérir davantage de notoriété. Ce n'est pas un hasard si la version finale contient des passages nouveaux, 
ayant une forte valeur politique et visant à inscrire l'auteur parmi les combattants de la cause révolutionnaire: il y souligne la nécessité, tant pour l'Italie que pour toute l'Europe, d'abattre, sous la poussée de la République française, toute sorte de «bastilles ", afin de permettre l'indépendance des peuples. Par exemple, si, dans les manuscrits, il se focalise essentiellement sur son parcours personnel, dans la version imprimée, il insiste en revanche davantage sur les objectifs politiques à atteindre pour "bâtir le grand édifice de la démocratie sur les ruines du despotisme, de la superstition, de l'ignorance $»^{11}$.

Ainsi, en publiant cet ouvrage à Milan à cette fin d'émancipation « universelle », son auteur (qui, jusqu'alors, n'avait été actif qu'en dehors de la péninsule) vise à faire connaître son passé révolutionnaire et ses convictions politiques dans un contexte nouveau. Son objectif principal est d'obtenir des charges dans ces institutions républicaines inédites et c'est pour ce motif qu'il se hâte d'envoyer plusieurs copies de son travail au directoire de la République cisalpine. De ce point de vue, il est fort emblématique que, quelques jours après la sortie des Memorie storiche, le ministre de la Police Gaetano Porro, véritable chef du courant radical cisalpin, profite de cette parution pour s'adjoindre les services de Rotondo, qu'il connaissait depuis quelques mois ${ }^{12}$. Le 9 août, il l'invite ainsi à se présenter le lendemain dans ses bureaux pour participer à la célébration organisée afin de «commémorer dans un banquet démocratique la journée du 10 août, sacre dans l'histoire de la liberté ${ }^{13}$ ». En effet, la présence de ce professeur est considérée comme fort utile, ayant personnellement vécu les événements de la journée parisienne de l'été 1792. De plus, deux mois plus tard, le ministre de la Police le charge officiellement de l'importante mission de «se rendre dans plusieurs endroits d'Italie afin de fraterniser avec les peuples libres». Ces fonctions attestent de la réussite de l'opération éditoriale, puisque c'est elle qui lance véritablement la carrière révolutionnaire de Rotondo en Italie ${ }^{14}$.

13 Cette décision de Porro démontre aussi la volonté de l'aile gauche du personnel politique italien d'étendre la révolution dans les autres territoires de la péninsule et, en même temps, de favoriser la participation des patriotes les plus radicaux, en les insérant dans les nouvelles structures républicaines. Mais surtout, cette charge - qui doit donc être menée non dans les bureaux fermés de l'administration cisalpine, mais dans les rues de l'Italie en révolution - confirme après coup la description que l'Argus Patriote, journal français dirigé par Charles de Morande, avait fait de Rotondo en juillet $1791^{15}$. Convaincu que «les étrangers surtout qui se permettent de nous endoctriner sont allés si loin qu'il est temps d'arrêter leur marche », celui-ci dénonçait la présence de nombreux étrangers en France et cherchait ainsi à restreindre l'acception du signifiant "patriote »: Morande avait inclus Rotondo dans la catégorie des réfugiés considérés comme dangereux et dont l'activité de propagande devait être arrêtée par la police ${ }^{16}$. Dans cette « secte Républicomaine » qui prêchait le « désordre en France », il n'y avait pas que des noms célèbres (comme Thomas Paine ou Anacharsis Cloots), mais aussi des profils moins connus, jugés peu doués d'un point de vue politique, mais d'autant plus à craindre à cause de leur influence sur le peuple. C'est le cas de Rotondo, qui est signalé avec un autre Italien, le diplomate Luigi $\mathrm{Pio}^{17}$, pour sa capacité à agiter les foules et à peser sur les hommes politiques les plus en vue. La description qui est faite de cet "agitateur » est fort emblématique de la réputation dont ce professeur italien jouit à Paris en ce début de Révolution et qui aide aussi à comprendre pourquoi, 
quelques années plus tard, le ministre cisalpin Porro le charge de cette mission auprès des autres peuples de la péninsule :

[...] Le sieur Rotondo n'est pas aussi fort en politique que M. Pio et il ne nous apprendra pas des choses aussi sublimes, mais il peut disputer avec avantage; il peut se plaindre, il peut crier, exciter l'intérêt et émouvoir la sensibilité de quelques membres des sociétés fraternelles. Il peut devenir un point de ralliement et servir de chef de file à des intrigants subalternes. Il ne sera jamais dangereux par luimême, mais ne pourrait-il pas, sans s'en douter, servir de machine ouvrière, à quelques-uns de nos grands réformateurs ? ${ }^{18}$

14 Ainsi, lors de la formation des "républiques sœurs" en Italie, la réputation que Rotondo s'est forgée en France - et lui a valu nombre de critiques et de problèmes - est déjà bien établie : elle va avoir des incidences décisives sur la suite de sa carrière. Il est donc nécessaire, à ce point de notre enquête, de se demander pourquoi cet homme, ce professeur de langues natif de Monza, avait été incarcéré aussi longtemps dans les geôles piémontaises avant de parvenir aussi rapidement, à Gênes puis à Milan, à gagner la confiance des chefs révolutionnaires de la péninsule. Bref, qui est Giambattista Rotondo et quelles ont été véritablement ses activités dans l'Europe en révolution?

\section{« Moitié » révolutionnaire, « moitié » aventurier}

Né le 29 janvier 1749 à Monza, Rotondo entre vite en conflit avec l'administration autrichienne, ce qui le pousse à démissionner en 1782 de ses fonctions de commis du bureau de l'Intendance royale de Milan, après avoir reçu à Vienne une réponse négative à sa demande de promotion. Il quitte ainsi le Milanais et commence à voyager dans le reste de l'Italie : il est à Reggio en Calabre quand, en février 1783, le tremblement de terre qui frappe cette ville le pousse à s'enfuir au-dehors de la péninsule. Il se réfugie donc dans la République des Provinces Unies, où il fréquente les milieux patriotiques de Rotterdam. C'est pour se soustraire aux perquisitions de la police qu'il est obligé un temps de se réfugier en Suisse, avant de déménager à nouveau pour s'installer à Bruxelles. Il est difficile ici de bien décrire les nombreux voyages qu'il entreprend à travers toute l'Europe. Même ses Memorie storiche n'aident guère à comprendre ses trajectoires, étant surtout riches d'imprécisions et d'omissions ${ }^{19}$.

Néanmoins, nous savons qu'en 1784, il est arrêté à Paris, après avoir été accusé d'avoir volé des montres d'or et des bijoux à des femmes de spectacle ${ }^{20}$. La détention ne dure que quelques semaines, car la dénonciation est vite révoquée, mais il est à souligner que Rotondo se garde bien d'évoquer cet épisode peu glorieux dans son autobiographie, dans laquelle il n'a pourtant pas hésité à raconter par le menu ses nombreux emprisonnements pour mieux se targuer du statut de persécuté. Treize ans après cette arrestation, lorsqu'il rédige ses mémoires en plein cœur du Triennio, son but est de se présenter comme un martyr exclusivement politique, totalement dévoué à la cause révolutionnaire: ainsi, tout ce qui n'est pas utile à la validation de cette autoreprésentation doit être soigneusement caché, d'autant plus qu'il s'agit en l'occurrence d'une affaire douteuse d'escroquerie.

17 En revanche, dans ses Memorie storiche il souligne avec force son adhésion précoce à la cause révolutionnaire : après la prise de la Bastille, alors qu'il travaillait depuis deux ans à Londres comme enseignant de langues, ce serait en apprenant la nouvelle de l'éclatement de « la révolution la plus extraordinaire que l'on a jamais vu en plusieurs siècles » qu'il aurait décidé de se rendre à Paris ${ }^{21}$. Dans la capitale française, il se lie 
rapidement avec le général Antoine Joseph Santerre, qui l'introduit dans les milieux sans-culottes $d u$ faubourg Saint-Antoine, et avec le futur conventionnel Léonard Bourdon, un des chefs de la prise de la Bastille, qui l'aide à ouvrir des cours en langue latine, italienne et anglaise dans les bureaux du Palais Royal ${ }^{22}$. À cette époque, il s'implique de plus en plus dans la révolution et vit un épisode singulier qui fait la «Une » des journaux parisiens: à la mi-novembre 1790, au cours d'une célèbre manifestation devant le château de Castries organisée en vue de fraterniser avec le député de l'Assemblée constituante Alexandre Lameth, il provoque, à la tête de ce cortège, le général Lafayette, intervenu pour réprimer les manifestants ${ }^{23}$. Interrogé par ce dernier sur son accent étranger afin de savoir s'il est Italien ou Anglais, Rotondo répond être "moitié l'un, moitié l'autre", sans manquer de faire allusion à l'origine nobiliaire de Lafayette (dont le nom de famille est du Motier), ainsi qu'à l'ambigüité du général à l'égard de la cause révolutionnaire ${ }^{24}$.

Nous ne savons pas si cet épisode est, comme Rotondo l'a présenté dans ses mémoires successifs, la source de la persécution dont il est victime dès lors de la part de Lafayette, mais le fait est que, durant cet automne, la police commence à diligenter des enquêtes sur son compte. Elle recueille plusieurs dépositions de citoyens témoignant l'avoir entendu tenir des « propos incendiaires » dans des lieux publics de Paris, comme le café du Palais Royal ou la terrasse des Feuillants. Ces témoins soutiennent avoir entendu ce professeur italien "dire à haute voix que la reine avait corrompu quarante-quatre députés pour conserver les ministres » et que « le roi ne se rassurerait plus de la leçon qu'on lui avait donné le 6 octobre 1789 et qu'il faudrait recommencer à en donner une ». Rotondo est décrit comme s'exprimant de la manière la plus injurieuse, car, « en se servant des épithètes et des qualifications les plus grossiers et les plus indécentes ", il aurait insulté la reine, la qualifiant de "putain, une malheureuse qui avait ruiné l'État», et le roi de "faute bête». Les témoignages affirment également que cet homme, ce "particulier que les uns disaient être Italien et les autres Anglais », aurait répandu « beaucoup d'horreurs contre $\mathrm{M}$. De Lafayette » et aurait même soutenu qu' « il fallait pendre tous les ministres sans exceptions». Finalement, il se distingue par des propos ouvertement violents: selon lui «il fallait encore que quelques têtes sautassent» ou encore le fait que "si on avait détruit la Bastille, on rétablissait Vincennes », où « la reine pourrait fort bien y être une des premiers renfermés » ${ }^{25}$.

19 Ainsi, début décembre, au bout de quelques semaines d'enquêtes, la police procède à son arrestation pour avoir tenu des "propos défavorables au Roi, contraires à la sagesse et aux bonnes mœurs de la Reine et à sa bonne conduite avec les ministres ${ }^{26}$. Or, peu de jours auparavant, Rotondo avait été victime d'un autre épisode mystérieux : alors qu'il se promenait en plein centre de Paris, il avait subi une agression à coups de bâton qui l'avait laissé presque sans vie. Les débuts de Rotondo en Révolution sont donc ceux d'un aventurier bien établi dans les hauts lieux de sociabilité populaire du Paris révolutionnaire, qui s'attire les suspicions de la police par ses propos exagérés et en même temps l'aversion des milieux délictueux de la ville. Mais il y a plus, car Rotondo arrive à mobiliser ces mésaventures pour acquérir toujours plus de notoriété dans les mouvements révolutionnaires, en faisant de sa situation privée une affaire publique. En effet, au cours des semaines suivantes, alors qu'il est emprisonné au Châtelet d'où il tente de se défendre, une grande partie des milieux parisiens, sans-culottes ou non, se mobilise en sa faveur ${ }^{27}$. 
20 Tout d'abord, son avocat ne se borne pas à la seule défense judiciaire, mais porte l'affaire sur un plan politique. Il utilise les discours injurieux que l'on prête à Rotondo comme une invitation à réfléchir, plus généralement, sur les problèmes relatifs à la liberté d'expression et au degré de protection publique dont peuvent jouir les autorités de l'État. Selon lui, l'affaire Rotondo «fait naître trois grandes questions d'ordre public » à l'égard de la nouvelle Constitution :

1. Quelle est la nature de propos personnels tenus en l'absence du chef suprême du pouvoir exécutif qui sont répréhensibles aux yeux de la loi, et quelle est la mesure de la punition?

2. Quels sont ceux punissables, à l'égard de l'épouse du Roi, des fonctionnaires publics, et quel est le genre de la punition?

3. Et enfin des propos vagues et inconsidérés, méprisables aux yeux mêmes de la société, quand ils ne sont pas provocatoires, précédés ni suivis d'actions ou attentats à l'ordre public [...] doivent-ils donner lieu à un décret rigoureux de prise de corps? La police civique ne doit-elle pas les punir paternellement? Ne serait-ce pas attirer toutes les haines et n'est-ce pas substituer le décret à la lettre de cachet?

Voilà les questions sur lesquelles l'accusé a besoin de lumières ${ }^{28}$.

21 Parallèlement, grâce à la médiation du général Santerre, l'affaire arrive au Club des Amis de la Constitution, dont les membres s'empressent de protester contre l'arrestation de Rotondo en obtenant de la part du député Adrien Duport la promesse de s'engager à pousser l'Assemblée à s'occuper d'une « loi d'habeas corpus perfectionnée sur celle des Anglais ${ }^{29}$. En outre, sous la pression de Santerre, qui est secrétaire du Club, les Amis de la Constitution excluent de leur sein Antoine Estienne, accusé d'être le chef de l'agression contre Rotondo ${ }^{30}$. S'ensuit un vif débat médiatique dans lequel, d'un côté, Estienne répond par un pamphlet, tandis que, de l'autre, l'Ami du Peuple de JeanPaul Marat et l'Orateur du Peuple de Louis Fréron défendent le professeur italien en utilisant cette affaire pour attaquer les proches de leur ennemi Lafayette ${ }^{31}$. Son arrestation pour les insultes au roi et à la reine est donc amplement relayée et instrumentalisée par la presse radicale, qui fait ainsi de son cas une cause d'intérêt général, tout en lui permettant de se faire un nom dans les milieux révolutionnaires parisiens $^{32}$.

Dès la mi-janvier 1791, Rotondo est libéré de prison et quelques mois plus tard, début avril, il est définitivement blanchi par un rapport jugeant autant «faux» qu'« extravagants » les propos qui lui ont été attribués ${ }^{33}$. Il en profite ainsi pour porter plainte contre le journal L'Ami de la Révolution ou Philippiques, qui l'avait accusé d'être un " homme dont l'existence est une injure à la société » et avait présenté sa libération comme une «espèce d'absolution qui annonce que les juges ont eu la conviction du crime, sans pouvoir réunir assez de preuves pour prononcer $»^{34}$. C'est ce dernier épisode qui lui permet de renforcer sa position au sein des milieux les plus radicaux de la capitale : non seulement il recommence alors ses cours de langues, mais surtout il entre officiellement au Club des Cordeliers, dont il signe plusieurs pétitions et où il parvient à conquérir l'estime de Marat ${ }^{35}$. En somme, Rotondo utilise à son avantage les persécutions qu'il subit et qui connaissent un écho retentissant par la publicité et l'instrumentalisation qu'en donne la presse radicale, pour se forger une réputation de pur martyr de la cause révolutionnaire et pour ainsi entrer en contact avec les milieux les plus avancés de la politique parisienne. Par exemple, début mai, il fait paraître une adresse Aux citoyens patriotes des sections de Paris dans laquelle il revient longuement sur ses déboires personnels et lie sa cause à celle «de tous les citoyens, de tous les 
étrangers $»^{36}$. De ce fait, il n'est pas étonnant qu'un an plus tard, son avocat - avec lequel entretemps il s'est également disputé - l'accuse devant les juges d'alimenter intentionnellement son affaire pour en tirer profit: « vous verrez que ses affaires sont mûres, en bon trait et prêts à juger, mais je doute que Rotondo veuille bien sincèrement en voir la fin... on ne parlerait plus de lui et il n'en parlerait plus aux autres $\aleph^{37}$.

Pas étonnant non plus qu'à l'été 1791, son nom soit inscrit sur la liste des étrangers dangereux qui est proposée par l'Argus Patriote. D'ailleurs, au moment précis où il est recensé comme tel par Morande, Rotondo est impliqué également dans les émeutes du 17 juillet, ce qui lui vaut une nouvelle arrestation en raison des propos incendiaires qu'il tient à l'encontre de la Garde nationale suite à la fusillade du Champ-de-Mars. Vers la fin de l'après-midi de cette fameuse journée, interpellant près du Pont-Neuf un groupe qui s'était rassemblé devant le café Conti, Rotondo se serait permis des "motions incendiaires contre la Garde Nationale », celle-ci « n'éta[n]t pas faite pour les empêcher de s'assembler». Ses propos entraînent l'intervention de la police du quartier, qui, en le jugeant " cause d'une extravagance dangereuse ", s'empresse de le conduire dans la prison de l'abbaye de Saint-Germain ${ }^{38}$.

Cette fois encore, sa détention ne dure que quelques semaines, bien qu'au cours de la perquisition de son appartement, la police découvre trois lettres compromettantes qui lui avaient été adressées au printemps précédent par Marat ${ }^{39}$. Une fois remis en liberté, il se hâte de faire paraître un autre pamphlet visant à dénoncer son arrestation et à renforcer encore un peu plus sa position au sein des milieux révolutionnaires. Il se décrit une fois encore comme un "martyr de la liberté ", comme un " homme traîné de cachots en cachots à cause de son patriotisme ", comme le "premier » à avoir donné «l'exemple en France de l'exercice du droit de responsabilité contre les abus du pouvoir et les attentats contre la liberté individuelle » (en clair, comme une victime de «l'ordre policier » de la monarchie constitutionnelle) ${ }^{40}$.

Néanmoins, son activité politique à l'étranger ne se borne pas à ces discours séditieux prononcés dans l'espace public. Elle s'articule de plus en plus étroitement aux menées politiques de plusieurs députés sous la Législative, ce qui lui vaut une ascension éphémère aux débuts de la République.

\section{2, ou un cas d'extrémisme sur le vieux continent}

À en croire la dénonciation du 5 brumaire an II (26 octobre 1793) que François Chabot prononce à son encontre à la tribune de la Convention, quelques temps après l'expulsion de Narbonne du ministère de la Guerre au printemps 1792, et alors que ce député siégeait encore à l'Assemblée législative, il fut contacté par Rotondo pour l'inciter à proposer une motion en faveur du renvoi aux frontières de l'ex-ministre. Faite à la veille de la déclaration de guerre, cette proposition vise à éloigner de Paris l'un des hommes les plus proches de Lafayette afin d'affaiblir la position politique de ce dernier et de s'emparer de l'argent de Narbonne. Cette offre fut refusée par Chabot elle aurait été par la suite réitérée à d'autres députés de l'aile gauche de l'Assemblée -, mais elle prouve en tout cas que ce professeur, qui s'ingénie à miner la réputation de Lafayette, a une réelle audience sur les milieux les plus influents de l'époque, puisqu'après avoir servi la cause du «triumvirat " en 1790 , il se rapproche sous la Législative de la nébuleuse « brissotine ${ }^{41}$. 
Mais le moment crucial de la carrière de Rotondo dans la France en révolution n'arrive qu'à l'été 1792 : alors qu'il est reconnu comme l'un des hommes les plus assidus aux réunions tenues au faubourg Saint-Antoine par le général Santerre ${ }^{42}$, il participe activement tant à la journée du 10 août qu'aux «massacres de Septembre ». Il y a là toutefois l'une des pages les plus obscures de sa carrière. D'après Georges Lenôtre, elle vaut à cet Italien, qui "auparavant faisait rire", de devenir, "pour tous ses contemporains, un objet d'horreur $»^{43}$. Le 7 septembre, il est chargé par la Commune de Paris d'une fonction autant importante qu'obscure : se rendre à Angers comme renfort des Commissaires «septembriseurs " Jean-Baptiste Félix et Joseph Bodson. D'après les recherches de François Uzureau, dès qu'il arrive dans la ville du Maine-et-Loire, muni d'une lettre de recommandation du Club central des Jacobins, Rotondo se charge de visiter les prisons en s'intéressant de près aux quelque 360 prêtres enfermés dans les bâtiments du séminaire : son intention aurait été de les faire tous fusiller et ce n'est que l'opposition de la municipalité locale qui aurait évité un tel massacre ${ }^{44}$. Le futur directeur Louis-Marie de La Révellière-Lépeaux, se trouvait lui-même à Angers le jour de l'arrivée des commissaires envoyés de Paris; il raconte dans ses Mémoires qu'« un étranger appelé Rotondo » était à la tête des agents envoyés dans l'Anjou par les « chefs de l'anarchie » et que ces «septembriseurs » eurent, dans le club de la ville, «si peu de succès, qu'à l'issue de la séance, on leur signifia que si le lendemain matin ils étaient encore dans la ville, ils seraient jetés dans la rivière $»^{45}$.

Rotondo est alors obligé de s'enfuir à Rouen, où son radicalisme lui vaut là encore d'entrer en conflit avec les autorités municipales ${ }^{46}$. Plusieurs de ses membres disent l'avoir « entendu prêcher une doctrine qui pouvaient occasionner des actions nuisibles à l'ordre et à la tranquillité publique qu'avait besoin [sic] cette Commune ». Quelques années plus tard, Rotondo admet, bien qu'à sa façon, les difficultés rencontrées à Rouen, avouant même que peu de jours après son arrivée, il avait été dénoncé à cause de ses "opinions politiques" puisque «les patriotes ne plais[ai]ent pas dans cette ville», au point qu'il aurait été forcé de se "cacher pour éviter la férule des aristocrates $\aleph^{47}$.

Or, comme à Angers, il parvient à s'échapper juste avant sa capture et décide alors de fuir la France pour rejoindre Londres à l'automne 1792. Certes, son séjour en Angleterre est d'abord le résultat de ses difficultés dans l'Hexagone, mais il nous semble également possible que ce transfert ait été sinon entièrement décidé, du moins canalisé par Paris. Dans son Mémoire pour le général en chef de l'Armée d'Italie rédigé à sa sortie des prisons piémontaises en 1796, il dit avoir " été expédié à Londres par Lebrun ministre, Danton, Condorcet et Vergniaud, pour surveiller les démarches du ministère britannique ${ }^{48}$. Si ses affirmations demeurent souvent peu fiables, cette circonstance s'avère en tout cas vraisemblable, d'autant plus que même la police sarde soutiendra, suite aux enquêtes menées pendant sa détention, qu'il «fut envoyé comme patriote en Angleterre où il avait déjà voyagé ". Ajoutons qu'à cette époque, il connaît bien l'Angleterre pour y avoir longtemps vécu (un avantage que n'ignore pas le ministère dans le choix de ses agents de renseignement) et qu'il est aussi en lien avec Condorcet, chez lequel - d'après les informations diligentées par les émigrés à Turin - il a eu ses entrées grâce à sa maîtresse Angélique ${ }^{49}$.

En tout cas, la police britannique, qui exerce d'emblée une surveillance étroite à son encontre, communique que ce "persecuted man » s'est réfugié à Londres «because in danger of his life in France », parce qu'il avait participé « at the murder of the prisoners 
from Orleans $\aleph^{50}$. Dans la capitale anglaise, Rotondo ne reste toutefois que quelques semaines, car, en étant désormais décrit par la police locale comme "a man more sanguinary than Marat $»^{51}$, il se voit obligé de se réfugier à Genève ${ }^{52}$. Mais là encore, c'est sa participation aux événements révolutionnaires de la ville qui lui vaut d'être à nouveau arrêté début avril. La nouvelle de son arrestation arrive vite en France, où Le Moniteur la communique par un passage qui ne fait qu'épaissir le mystère sur la teneur de ses activités révolutionnaires et sur la sincérité de son engagement politique :

Une lettre de Genève porte qu'un fameux agitateur de Paris, nommé Rotondo, qui, à l'aide de deux ou trois cents brigands, devait exécuter un pillage et massacrer les membres de tous les comités, vient d'être arrêté. On a trouvé chez cet homme une forte somme d'assignats et 20.000 livres en espèces. Il a, dit-on, déjà promis aux guichetiers de faire leur fortune s'ils favorisaient sa fuite... ${ }^{53}$

31 Cette fois, pourtant, sa détention dure bien plus que quelques semaines, puisqu'elle ne prend fin qu'au bout de trois ans et demi. En effet, après avoir été transféré d'abord dans le canton de Berne, puis dans le Piémont, Rotondo n'est libéré qu'en décembre 1796, en conséquence de l'entrée des armées républicaines en Italie. Arrivé à ce point $\mathrm{du}$ récit du parcours de Rotondo, encore faut-il souligner que l'expérience « révolutionnaire » de ce professeur se situe avant la prise du pouvoir des montagnards du 31 mai 1793 et s'est essentiellement faite, en France, sous la monarchie constitutionnelle. Bien que sans doute l'une des plus radicales parmi celles des Italiens réfugiés en France, cette expérience-ci n'est donc en rien liée à la période de la Terreur ${ }^{54}$.

32 En tout état de cause, sa détention se caractérise par des intrigues politiques liées à son activité parisienne précédente. Par exemple, son déménagement dans le château de Chillon, près de Nyon, se fait sous la pression des émigrés français et anglais, qui le décrivent aux autorités du canton de Berne "comme un scélérat qui eut égorgé les suisses leurs compatriotes dans la journée des Tuileries du 10 août $1792 »^{55}$. Quant à son transfert ultérieur dans les prisons du Piémont, qui a lieu début septembre, Le Moniteur du 19 de ce mois, anticipant en quelque sorte la version de l'« intrigue politique » que Rotondo reprendra lui-même à son compte dans ses mémoires ${ }^{56}$, publie la transcription d'une lettre où ce transfert en Piémont est décrit comme un " prétexte » utilisé par les troupes suisses afin de s'unir aux armées sardes et attaquer les territoires français ${ }^{57}$.

Mais si ce professeur est extradé dans les prisons du royaume sarde, c'est surtout en raison de la volonté des Suisses de s'en débarrasser en le bannissant, mais c'est aussi du fait des instances réitérées du gouvernement de Turin, qui réclame depuis longtemps le droit de pouvoir le juger. Dans le Piémont, Rotondo est surtout honni pour être l'auteur supposé de l'homicide de la princesse de Lamballe, l'amie célèbre de la reine MarieAntoinette, née à Turin et morte à Paris au cours des massacres de septembre. Ce professeur de langues paye donc encore une fois son activité révolutionnaire de l'été 1792, mais cette fois-ci, ces imputations ont une origine d'une toute autre envergure. C'est en fait Jacques Mallet du Pan, à l'époque demeurant en Suisse, qui fournit à la cour de Turin les renseignements sur Rotondo - renseignements qu'il tient d'un autre émigré contre-révolutionnaire célèbre, l'ex-ministre de la Marine Antoine Bertrand de Molleville. Celui-ci, depuis Londres, lui avait adressé plusieurs témoignages relatifs aux discours prononcés par cet Italien lors de son exil en Angleterre, où il se serait fait une gloire dans les cafés d'avoir tué lui-même la princesse de Lamballe et d'en avoir porté la tête au bout d'une pique ${ }^{58}$. Même s'il est impossible de confirmer la validité de cette information ou de rendre compte de l'évolution du procès mené par le Sénat 
piémontais, il est significatif que les nouvelles du meurtre de la princesse de Lamballe se répandent moins en France qu'à l'échelle européenne: cela montre en fait que, même du côté contre-révolutionnaire, une véritable structure communicative visant à utiliser à son avantage les événements français est mise en place dans un vaste espace continental ${ }^{59}$.

Dans le château d'Ivrée, situé dans le Piémont septentrional, Rotondo, soumis à un régime alimentaire drastique, se réfugie dans la lecture d'auteurs comme Fénelon et Montesquieu, dont il parvient à soustraire les ouvrages à la surveillance de la police. Il se consacre également à la rédaction de quelques réflexions politiques sur la fonction révolutionnaire de la guerre ${ }^{60}$. Ce n'est qu'à l'issue du dernier épisode de cette guerre, celle menée dans la péninsule par les armées républicaines à partir du printemps 1796 que, le 13 décembre, il est finalement libéré. Il peut alors commencer sa bataille personnelle contre les gouvernements responsables de sa détention afin de se faire rembourser des pertes subies, mais il lui est surtout permis de reprendre une activité politique, à ceci près que celle-ci se fait désormais dans sa propre patrie.

\section{Dans I'Italie du Triennio : une action politique de courte durée}

Libéré par les armées républicaines en plein cœur du Triennio, ce professeur de langues, qui s'est illustré dans les phases les plus critiques de la Révolution française, cherche d'emblée à s'impliquer dans le processus de démocratisation qui s'amorce en Italie. Si une grande partie des patriotes de la péninsule s'active pour soutenir sa collaboration dans les nouveaux réseaux républicains, c'est parce qu'en fin des comptes, Rotondo apparaît comme le prototype du "parfait révolutionnaire » : celui qui a participé à la «Grande Révolution » et a longtemps souffert en prison pour sa fidélité et sa constance aux «bons» principes politiques. À l'été 1797, une fois ses Memorie storiche parues, Rotondo apparaît en effet aux yeux des patriotes de la péninsule - qui ignorent évidemment tout de ses excès et ses ambigüités - comme «le vieil ami» des révolutionnaires de Paris tels que Marat et Santerre; "l'Italien de France " ayant activement contribué à la chute de la monarchie au-delà des Alpes; le "persécuté " recherché à travers toute l'Europe parce qu'il avait « le républicanisme dans le cœur et dans la langue $»^{61}$.

Ce n'est donc pas un hasard si le ministre de la Police Porro se hâte de l'inviter à la fête organisée pour célébrer l'anniversaire de la journée du 10 août, puis le charge de la fonction de "se rendre dans plusieurs endroits d'Italie afin de fraterniser avec les peuples libres ». Rotondo a réussi, par ses écrits et ses réseaux, à mettre en valeur son expérience dans l'Europe en révolution, en renversant positivement la description que l'Argus Patriote avait fait de lui : s'il n'est pas cet homme particulièrement «fort en politique ", il est clairement capable de "servir de machine ouvrière » pour certains "grands réformateurs". Une fois revenu sur sa terre natale, il peut à ce titre contribuer à la diffusion de la révolution dans la péninsule.

Voyageant dans les villes italiennes sous le titre de Commissaire républicain dès le mois de novembre 1797, il se rend dans un premier temps dans les territoires de l'exRépublique cispadane (notamment à Modène et Bologne), puis en Toscane. C'est là, qu'à la faveur des recommandations de son protecteur Porro, il est reçu à Livourne par 
l'ambassadeur cisalpin Alessandro Sacerdoti, puis à Lucques, où il est signalé comme œuvrant à révolutionner cette ville pour favoriser son annexion à la Cisalpine ${ }^{62}$. En février 1798, une fois la République romaine proclamée, il décide de rejoindre Rome, où, un mois plus tard, il échafaude un plan ambitieux : il propose de se rendre à Naples afin de nouer des contacts avec les patriotes méridionaux et préparer ainsi l'invasion militaire de ce royaume et le renversement de la monarchie de Ferdinand IV de Bourbon. Malgré sa décision d'agir incognito, il est vite découvert par des espions à la solde de la Cour. D'après le récit qu'il communique quelques semaines plus tard à deux importantes autorités de la République cisalpine qui sont entretemps devenus ses protecteurs, Carlo Testi (ministre des Affaires Étrangères) et Giuseppe Abamonti (secrétaire du ministère de la Police), «bien qu'[il ait] tout essayé et tout mis en œuvre afin de surprendre la vigilance du tyran napolitain et de sa femme digne de lui (la Messaline autrichienne), tout a été inutile, car à présent il y a peut-être plus d'espions à Naples que dans toute Europe $»^{63}$.

Secrètement aidé par quelques patriotes de la ville, il est alors obligé de s'enfuir du Royaume et de se réfugier à Livourne, d'où, fin-juin, il écrit à ses responsables pour les informer de sa décision de démissionner et de repartir pour Paris pour y reprendre sa profession de professeur de langues ${ }^{64}$. De fait, la situation politique du gouvernement cisalpin a entretemps beaucoup changé et l'éviction du ministre Porro lui a valu la perte de son principal protecteur. En outre, l'imposition à Milan d'un traité d'alliance avec la France réduisant fortement l'autonomie d'action de la République cisalpine en matière de politique étrangère et la création à Rome d'une République subordonnée au Directoire ont augmenté l'opposition au gouvernement français des milieux les plus radicaux.

En Italie, Rotondo n'a donc pas réussi à pérenniser sa collaboration avec les institutions républicaines, ce qui confirme encore la singularité de son cas. Ceci prouve que, en dépit du crédit dont il jouit initialement en vertu de son expérience révolutionnaire, celle-ci ne suffit pas à lui faire jouer un rôle politique notable et durable dans son propre pays.

Si cette courte période d'action au service de la République cisalpine est parfaitement révélatrice des excès de sa personnalité et de son radicalisme politique, elle ne doit pas cependant être considérée comme la preuve du virage foncièrement modéré du gouvernement milanais dans son ensemble. À l'été 1797, l'attention qu'un ministre aussi important que Porro porte à un "extrémiste» comme Rotondo prouve en fait qu'au moment de la naissance de la République cisalpine, tous les scenarios politiques étaient envisageables et toutes les solutions institutionnelles possibles. Le soutien dont une «tête exaltée » comme la sienne jouit au cours de cette phase montre la volonté initiale du courant patriotique italien de favoriser l'introduction dans ses structures républicaines inédites des hommes les plus radicaux, ce qui mérite d'être considéré comme une tentative pour fortifier la position de cette « république sœur » à l'égard du Directoire parisien, en renforçant son autonomie et en initiant une politique unitaire envers les autres États de la péninsule. En même temps, le profil de Rotondo et l'échec de son entreprise prouvent aussi, a contrario, que la lutte politique de l'Italie du Triennio est bien davantage inscrite dans les vicissitudes de la période directoriale qu'en regard des premières années de la Révolution ${ }^{65}$.

Reste que l'engagement politique de Rotondo est loin de s'interrompre : il continue à afficher ouvertement ses convictions radicales et n'hésite pas à critiquer Bonaparte, 
quand bien même ce dernier eût permis sa libération. Par exemple, lors de son passage à Nice à la mi-juin 1798, le Commissaire de la ville se hâte d'écrire au ministre de la Police, Le Carlier d'Ardon, pour l'informer que seulement «quelques heures de conversation" avec cet Italien lui ont suffi pour dévoiler un "homme à grand caractère, entreprenant, exalté, apôtre et martyr de la liberté, mais paraissant n'avoir pas la meilleure opinion du vainqueur de l'Italie ${ }^{66}$.

De retour à Paris presque six ans après sa fuite précipitée de France à l'automne 1792, il est encore une fois arrêté, ce qui achève de confirmer sa compromission dans les massacres de Septembre. Informée de son arrivée dans la capitale par les plaintes de la maitresse de l'hôtel où, depuis quelques jours, Rotondo logeait sans payer son loyer, la police parisienne le conduit en prison fin juillet, avant qu'on ne lui intente un procès dont le chef d'inculpation n'a rien à voir avec les griefs de la maitresse de l'hôtel, mais qui est en revanche clairement lié à son activité politique passée. Après avoir entamé leur enquête sur son compte, les procureurs notent que, dès le « commencement de la Révolution, il s'y est prononcé dans le genre révolutionnaire dans la manière la plus atroce » et que lorsqu' « il s'est trouvé compromis, il a fui de Rouen pour se soustraire à un mandat lancé contre lui ${ }^{67}$. La suite du procès confirme que la vraie cause de son arrestation est bien antérieure à son retour à Paris, car, au bout de nombreux interrogatoires et de l'analyse attentive de ses papiers faite par deux traducteurs italiens, le bureau central de Police estime que «Rotondo a été arrêté comme UltraRévolutionnaire, ou plutôt comme agent du gouvernement anglais sous les formes d'un patriotisme exagéré». Les rapporteurs signalent aussi que, bien que Rotondo ait "produit des passeports et des pièces honorables ", il apparaît que " cet homme n'est pas aimé et qu'en effet il avait tenu, depuis le commencement de la Révolution française, une conduite si non criminelle, au moins peu conforme aux principes de sagesse et de modération qui dirigeaient le gouvernement ». Et d'en conclure que " cet homme singulier, dont l'existence est étonnante [...], paraît effectivement avoir une tête exaltée $»^{68}$.

Néanmoins, les inspecteurs ajoutent qu'en l'absence de preuves concrètes de sa culpabilité, ils ne peuvent pas prolonger la détention de Rotondo en France et que « tout ce qu'on peut arrêter [est] qu'il quitte le territoire de la République » et qu'il soit retenu «dans son Pays sous surveillance des autorités ». En effet, même les personnes chargées d'examiner ses papiers saisis ont déclaré n'avoir «rien trouvé qui puisse donner lieu au moindre soupçon, mais bien des preuves multiples et irréfutables de son civisme et de son brûlant amour pour les républiques en général et pour la République française en particulier»; d'après eux, ces documents "ne contiennent absolument rien qui puisse faire élever des soupçons contre lui ", mais "sont plutôt autant de preuves de son patriotisme ardent et éclairé ${ }^{69}$. Ainsi, fin août, alors que la situation politique de la République cisalpine est au centre du débat français à cause du projet de réforme de la Constitution préparé à Milan par l'ambassadeur Charles-Joseph Trouvé ${ }^{70}$, en dépit des demandes adressées par Rotondo à Paul Barras pour être autorisé à rester en France, le Directoire ordonne sa déportation définitive hors du territoire de la République $^{71}$.

Il est toutefois remarquable que la nouvelle de son arrestation, vite arrivée à Milan, ait suscité autant de craintes de la part des politiciens cisalpins, qui craignent des répercussions dans les équilibres de cette "République sœur", étant conscients que quelques mois auparavant, Rotondo y a bénéficié de nombreuses protections parmi le 
personnel politique évincé. Par exemple, à la veille du coup d'État milanais de Trouvé (qui a lieu le 31 août 1798), le poète Vincenzo Monti, tout en étant bien loin de partager le radicalisme de Rotondo, écrit à son ami Giovanni Costabili Containi, ex-membre du directoire cisalpin, pour lui communiquer son inquiétude au sujet de cette arrestation, non pas parce qu'il connait directement le professeur de Monza, mais parce qu'il interprète cette nouvelle comme compromettante pour l'ex-protecteur de Rotondo, Carlo Testi, qui est alors membre du directoire et de qui Monti avoue recevoir des faveurs ${ }^{72}$.

45 Au même moment, le ministre des Affaires étrangères Ambrogio Birago confie lui aussi à son fonctionnaire à Paris Francesco Visconti Aimi la délicatesse d'une telle affaire, en confirmant non seulement la réputation négative dont Rotondo jouit à Milan, mais aussi et surtout les protections nombreuses que, lors de la naissance de cette République sœur, cet homme avait gagnées au sein du gouvernement cisalpin :

Je dois vous observer que cet individu avait des relations avec la majorité des membres du gouvernement lors de sa formation, mais qu'après il a été regardé comme un scélérat, un homme atroce et pervers jusqu'à être éloigné d'une commission d'espionnage dont la police le chargeait. Se voyant l'objet d'un mépris universel, il s'est absenté et notre police s'est faite un devoir de prévenir le gouvernement des lieux où il allait, comme à ceux de Toscane et de Gênes, pour désavouer ses opérations et le peindre comme le rebut des hommes ${ }^{73}$.

Bref, derrière les charges retenues à l'été 1797 à l'encontre de ce professeur impliqué dans plusieurs révolutions européennes, se sont joués des enjeux politiques de premier plan, dont témoigne l'inquiétude de plusieurs acteurs politiques encore en fonction, qui cherchent alors à se désolidariser de sa "cause». Ce sont ces volte-face ou, plus exactement, cette disgrâce prononcée par le personnel politique cisalpin qui ont causé la sortie définitive de Rotondo de la vie publique, tant en Italie qu'en France.

\section{Une disparition brutale et une anecdote mystérieuse}

S'il n'est en rien étonnant qu'un homme comme Giambattista Rotondo, inlassablement discrédité comme une "tête exaltée » tout au long de la décennie révolutionnaire, ne puisse escompter aucun retour en grâce sous Napoléon, il faut toutefois souligner que son cas démontre à quel point le retour dans le pays d'origine d'un étranger en révolution n'est pas sans incidence sur les équilibres politiques locaux, sans être pour autant synonyme de responsabilités pérennes. Tout en étant influencées par le contexte international, les dynamiques révolutionnaires de chaque pays répondent à des logiques multiples, conditionnées par l'évolution de la lutte politique nationale et par les particularités de leurs acteurs locaux. De ce fait, ces dynamiques ne peuvent être considérées comme le strict décalque ou la répercussion directe d'autres processus.

Tout en permettant une lecture résolument transnationale de ces dynamiques politiques, la perspective des "révolutions croisées" invite à enquêter sur les influences réciproques entre plusieurs processus révolutionnaires, dans la mesure où il s'agit de refuser des approches proposant un rayonnement à sens unique, d'un pays vers un autre. Réévaluer l'originalité de chaque processus ne signifie pas l'évincer d'un contexte international, ni nier les influences qu'il reçoit d'autres modèles, mais témoigne de la nécessité de bien mettre en évidence les spécificités de ces dynamiques, en soulignant tant leurs analogies que leurs spécificités intrinsèques. Dans cette 
logique, les mobilités révolutionnaires (et contre-révolutionnaires) s'avèrent un champ d'étude foisonnant, toute circulation engendrant des transferts remarquables. Cependant, elles ne doivent pas simplement être perçues comme un vecteur automatique de rayonnement exercé par un modèle politique sur un autre, tant il est vrai que toute bataille politique nécessite d'être replacée dans son contexte et dans son époque. Comme le montre le cas de Rotondo, les protagonistes de ces dynamiques migratoires n'arrivent pas forcement à imposer leurs pratiques dans le pays d'origine et cela d'autant moins que leur présence à l'étranger apparaît aussi riche de potentialités que de conflits et ne doit pas occulter non plus leur part d'ambiguïté et d'instrumentalisation réciproques. Ces réfugiés se montrent en fait surtout capables d'utiliser les persécutions qu'ils subissent comme un formidable levier pour se forger un statut de « martyrs » et pour étoffer ainsi leurs réseaux de protecteurs politiques.

Quant à la suite du parcours de ce si étrange professeur italien, les traces se font plus sporadiques et presque toujours en lien avec des activités exagérées qui lui valent de nouvelles arrestations. En mars 1802, quelques semaines après la naissance officielle de la République italienne décrétée par la Consulte de Lyon, Rotondo est expulsé encore une fois de France, où il était entretemps retourné ${ }^{74}$. Les institutions consulaires le signalent alors comme l'un des « chefs de faction [...] aux ordres de l'Angleterre » qui, «n'ayant point d'autre objet que celui d'exciter les troubles, se trouvaient dans toutes les émeutes $\aleph^{75}$. Un an plus tard, on le retrouve à Rome, où il est dénoncé par le diplomate François Cacault comme l'un des fondateurs d'un «Club anglais » avec des émissaires de la reine de Naples ${ }^{76}$. En 1811, il est une fois encore arrêté après avoir été accusé d'avoir commis un "vol à main armée " sur les routes du Piémont, ce qui tendrait à montrer que son extrémisme a fini par le réduire, à l'époque impériale, à une marginalité totale qui le fait sombrer dans le brigandage $\mathrm{e}^{77}$.

Dix ans plus tard, en 1822, alors qu'en Italie vient de s'achever un nouveau tournant révolutionnaire avec la « révolution constitutionnelle » de 1821, Rotondo est à nouveau épinglé à Paris pour son passif révolutionnaire. Madame Campan, l'ancienne première femme de chambre de Marie-Antoinette, rend publique une information qui confirme les soupçons élevés à son encontre lors de son conflit avec La Fayette. Dans un passage de ses Mémoires, dont la fiabilité reste toutefois douteuse, elle décrit une tentative d'assassinat de la reine de la part de l'aventurier italien qui aurait eu lieu le soir du 14 juillet 1790 :

On se rendit à Paris pour la première fédération, le 14 juillet, anniversaire de la prise de la Bastille. [...]. La cour revint à Saint-Cloud après la fédération: un scélérat, nommé Rotondo, s'y introduisit dans le dessein d'assassiner la reine. On a su qu'il avait pénétré jusque dans les jardins intérieurs: la pluie empêcha Sa Majesté de sortir ce jour-là. M. de La Fayette, qui avait eu connaissance de ce complot, donna les consignes les plus sévères à tous les factionnaires; et le signalement de ce monstre fut répandu dans le palais, par l'ordre du général. J'ignore comment on parvint à le soustraire au supplice ${ }^{78}$.

51 Jusque sous la Restauration, la réputation sulfureuse que ses excès ont valu à Rotondo continue donc à alimenter tous les fantasmes. Si difficiles qu'ils soient à dissiper, il n'en reste pas moins que son parcours chaotique dans l'Europe des Révolutions mérite d'être tiré de l'oubli, par ce qu'il révèle des significations et usages pluriels de l'engagement radical de ces étrangers. 


\section{NOTES}

1. Giambattista Rotondo, Memorie storiche del professore Gio. Bat. Rotondo, cioè delle avventure più rimarchevoli della sua vita dall'anno 1782 fino al 1796 inclusivo, scritte da lui medesimo, Milan, Civati, 1797. Une copie de cet ouvrage est conservée aux Archives nationales de France (dorénavant AN) sous la cote F/7, carton 6155, dossier 1133, pl. 2. Classé sous le nom «Affaire Rotondo Demouza », ce carton contient de nombreux documents concernant le parcours politique de Rotondo qui ont nourri le présent travail. Je tiens à remercier Antonino De Francesco de m'avoir suggéré cette source précieuse et de m'avoir encouragé à enquêter sur ce personnage.

2. Giornale de' Patrioti d'Italia, n. 80, 4 thermidor an premier de la liberté italienne (22 juillet 1797).

3. À ce propos, voir Antonino DE FRANCESCO, «La Révolution française hors de France : quelques perspectives de recherche sur l'historiographie italienne entre le $\mathrm{xIX}^{\mathrm{e}}$ et le $\mathrm{xx}^{\mathrm{e}}$ siècle », AHRF, $\mathrm{n}^{\circ}$ 334, 2003, p. 105-118; Eugenio DI RIENzo, «L'histoire de si' et l'histoire des faits'. Quelques perspectives de recherche à propos de l'historiographie italienne sur la période révolutionnaire, 1948-2000 », AHRF, n 334, 2003, p. 119-138.

4. Armando SAITTA, «Il robespierrismo di Filippo Buonarroti e le premesse dell'unità d'Italia ", Belfagor, vol. 10, n³ 3, 1955, p. 267-270. Du côté français, cette lecture a été relancée par Jacques GODECHOT, «Les Jacobins italiens et Robespierre », AHRF, n 30, 1958, p. 65-81. Quant aux fortunes des études sur Buonarroti dans l'historiographie italienne de l'après-guerre, voir Antonino DE FRANCESCO, «L'ombra di Buonarroti. Giacobinismo e rivoluzione francese nella storiografia italiana del secondo dopoguerra ", dans ID., Mito e storiografia della "Grande Rivoluzione » nella cultura politica italiana del '900, Naples, Guida, 2006, p. 341-376.

5. Filippo BUONARROTI, Conspiration pour l'égalité, dite de Babeuf, suivie du procès auquel elle donna lieu et des pièces justificatives, les deux premières éditions sont parues à Londres et à Bruxelles en 1828, alors qu'une troisième version a été publiée à Paris en 1830 par les frères Baudouin. Sur l'activité de Buonarroti en France sous la Révolution, et notamment sur sa charge de Commissaire républicain à Oneille pendant la Convention, voir Paolo CONTE, «Le Commissariat d'Oneille : audelà de Buonarroti (1794-1796) », AHRF, nº 388, 2017, p. 75-105.

6. Pour une présentation élargie du devenir des Italiens en France sous la Révolution, voir Anna Maria RAO, Esuli. L'emigrazione politica italiana in Francia (1792-1802), Naples, Guida, 1992.

7. «Mon cher Général, le citoyen Faypoult, ministre de la République française à Gênes, vient de me recommander particulièrement le citoyen professeur Rotondo, Milanais, naturalisé français et ci-devant établi depuis 15 ans à Paris, comme vrai et zélé patriote. Par ce digne titre et par la recommandation de l'ami Faypoult, je me vois engagé à lui accorder ce qu'il me demande et que je trouve très juste. Il réclame office auprès de vous, mon cher Général, afin que vous lui accordiez la grâce de lui fournir une lettre de recommandation auprès du Roi de Sardaigne, qui le fît arrêter en Suisse et gémir dans les prisons du Piémont pendant trois ans et dix mois [...]. Un seul mot de votre part, mon cher Général, suffira à ce pauvre et martyr de la Révolution française pour obtenir du Roi de Sardaigne ce qu'il a droit de réclamer ». AN, F/7, carton 6155, dossier 1133, pl. 11.

8. Carizzi à Salfi, v. fin mars 1797 : « La presente ti sarà esibita dal cittadino Rotondo. Questi è una di quelle vittime del dispotismo sardo: dopo una lunga e penosa prigionia ha avuto il piacere, non si sa come, di respirare e veder di nuovo quella luce che per anni eraglisi interdetta. Si porta ora nel suolo della libertà. Ora, sicuro dalle insidie e dagli artigli della tirannia, vuol pubblicare all'universo l'ingiusta ed orrenda persecuzione sofferta pel despotismo del più vile de' tiranni. Ha bisogno della tua penna e della tua maschia eloquenza, non perché la verità avesse bisogno de' 
colori per farsi strada, ma per metter in piena veduta gli orrori dell'arbitrario potere. Te lo raccomando con quel calore che merita un amico della buona causa, un seguace della Libertà. [...] La tua penna è più distruttiva e micidiale pe' despoti che l'attuale spada de' repubblicani. Gli affari prendono sempre più felice aspetto nella nostra Italia. Alla più orrenda tempesta succede per una volta la consolante calma pel genere umano! Lo spero, e ne sono anzi sicuro. Viva la libertà. Viva la Repubblica italiana. Viva la felicità e il riscatto dell'Universo ». AN, F/7, carton 6155, dossier 1133, pl. 1.

9. Ibid., pl. 7.

10. Parmi les documents qui sont confisqués à Rotondo par la police parisienne en août 1798 et qui, aujourd'hui, sont conservés aux AN sous la cote F/7, carton 6155, dossier 1133, il existe trois manuscrits des Mémoires historiques, auxquels a été ajoutée la version imprimée, également conservée dans ce dossier. Une première version, se composant de 46 feuilles, est rédigée en français et à la première personne ; un deuxième manuscrit de 51 feuilles est également à la première personne, mais en italien ; la dernière version, comptant 43 feuilles, est écrite en italien et à la troisième personne. C'est à cette troisième mouture du texte que la version imprimée est la plus fidèle.

11. Giambattista ROTONDO, Memorie storiche..., op. cit., p. 65-66.

12. Les rapports entre Porro et Rotondo sont attestés par un passage d'une lettre écrite par ce dernier à son cousin, le futur membre du directoire cisalpin Fedele Sopransi : «Parmi les connaissances et amis que j'ai encore trouvés à mon retour à Milan, j'ai eu satisfaction de voir très souvent le citoyen Porro, membre de l'administration Lombarde, qui jouit de la plus haute estimation publique. Il est de vos amis et veut bien s'unir à moi pour vous recommander, mon cher cousin, ma malheureuse affaire ». AN, F/7, carton 6155, dossier 1133, pl. 7.

13. Ibid., pl. 10.

14. Ibid., pl. 9.

15. Pour une analyse de ce journal et de la biographie de son auteur, voir Simon BURRows, A King's Ransom. The Life of Charles Théveneau de Morande, Blackmailer, Scandalmonger et Master-Spy, Londres, Continuum, 2010.

16. Tout au long de l'été 1791, dans un Paris encore sous le choc de la fuite du roi, Morande revient à plusieurs reprises sur les activités des étrangers en France, dénonçant moins leurs origines que leur activité politique. D'après lui, la présence de ressortissants d'autres pays doit être considérée comme une source non-négligeable de préoccupations, d'autant plus que ces citoyens sont en mesure d'influencer la vie politique de leur pays d'accueil. Il refuse en fait toute forme d'engagement de ces étrangers qui puisse se faire par le biais de leur participation aux sociétés patriotiques et soutient que cette présence en France s'inscrit dans un projet plus vaste mené par les « ennemis du dehors » visant à augmenter le désordre politique de l'intérieur. Voici un des passages les plus intéressants parus pendant l'été 1791 : «Dans tous les cas, quoique M. Ephraim, madame la Baronne d'Aelders, M. Brown Dignam, M. Pio, M. de Clavières et le vertueux Anacharsis puissent nous dire ; tant que nous ne serons pas rendus à un état de tranquillité, les étrangers ne doivent pas être admis dans les sociétés patriotiques, surtout dans celles qui forment des arrêtés, qui prennent des résolutions et qui envoient des adresses aux sociétés qui leur sont affiliées. Quand les étrangers seraient tous honnêtes (et il faut faire des exceptions), ils peuvent tous être soupçonnés d'avoir des intérêts à défendre et de former des vœux différents des nôtres. Pourquoi serions-nous si étonnement généreux envers les fugitifs étrangers qui résident parmi nous, puisque les Français qui habitent chez les Nations étrangères ne sont admis dans aucune de leurs sociétés patriotiques? ». L'Argus Patriote, $\mathrm{n}^{\circ}$ 16, 2 août 1791, p. 419-420.

17. Sur Pio, voir Albert MATHIEZ, "La Révolution et les étrangers. Le chevalier Pio », Annales révolutionnaires, $\mathrm{n}^{\circ}$ 11, 1919, p. 94-104. Des informations plus récentes et fort intéressantes sur son compte sont dans Virginie MARTIN, «La diplomatie en Révolution. Structures, agents, pratique et 
renseignements diplomatiques : l'exemple des agents français en Italie (1789-1796) ", thèse de doctorat soutenue à l'université Paris 1 Panthéon-Sorbonne en 2011, t. 2, p. 452-471.

18. L'Argus Patriote, $\mathrm{n}^{\circ} 9,7$ juillet 1791, p. 219.

19. Après avoir travaillé sur la carrière de Rotondo, Georges Lenôtre décrit les mémoires de ce professeur italien comme un «recueil de hâbleries et de mensonges, écrit pour dérouter une enquête possible » et soutient qu' «il faut donc faire une abstraction presque complète de son récit ». Georges LENÔTRE, «Rotondi-Rotondo, professeur de langues », dans ID., Vieilles maisons, vieux papiers, t. 2, Paris, Perrin, 1961, p. 144-145.

20. Les dossiers concernant cette arrestation sont en AN, Y, carton 9942 ; AN, F/7, carton 4625, f. 117-119.

21. Giambattista ROTONDO, Memorie storiche..., op. cit., p. 17-18.

22. F/7, carton 6155, dossier 1133, pl. 7, 10.

23. Les Révolutions de Paris, $\mathrm{n}^{\circ} 75,11$ décembre 1790.

24. Sur cette affaire, voir aussi Georges LENÔTRE, « Rotondi-Rotondo... », art. cité, p. 129-149.

25. AN, Y, carton 13320 ; AN, F/7, carton 4625, f. 191.

26. AN, Z/3, carton 1, Extrait sur l'affaire criminelle du S. Rotondo, fait sur les pièces étant au greffe du Chatelet.

27. Dans son interrogatoire du 9 décembre, Rotondo reconnaît avoir prononcé des discours politiques, mais affirme qu'il a été mal compris en raison de sa mauvaise maîtrise du français. Il admet en effet « qu'il est cependant possible qu'entendu sa difficulté de s'exprimer en français et qu'en prenant très souvent l'occasion de rendre sa féconde pensée sur le gouvernement d'Angleterre et se supposant transporté à Londres où réside la vraie liberté, il lui soit échappé des propos politiques et pleinement permis en Angleterre sur le compte du Roi, de la Reine et des Ministres de cette île et que cela n'est mal interprété par ceux qui les auront entendus et qui les auront appliqués au Roi et à la Reine de France ». AN, F/7, carton 4625, f. 134.

28. AN, Z/3, carton 1, Extrait sur l'affaire criminelle du S. Rotondo, fait sur les pièces étant au greffe du Chatelet.

29. Ibid.

30. AN, F/7, carton 4625 , f. 137 , Copie du procès-verbal de la séance par les amis de la Constitution, vainqueurs de la Bastille. Le texte est signé par Santerre en tant que secrétaire et adressé à l'Ami du Peuple.

31. Antoine ESTIENNE, Dénonciation des libelles intitulés : l'Ami du peuple par Marat et l'Orateur du Peuple par Martel, et réflexions sur la liberté de la presse, 1791 (BnF, coll. LB39-5213). L'auteur se défend des accusations d'agression contre Rotondo et en propose une toute autre interprétation: «J'ai appris depuis que Rotondo ayant [...] insulté un Garde National, on lui avait proposé de se battre en duel, et qu'il avait refusé ; d'après ce refus, on lui avait promis des coups de bâton ». En 1791, l'affaire Rotondo est traitée surtout dans les numéros 301, 306, 338, 342, 347 de l'Ami du Peuple et dans les numéros 4, 11, 32, 38, 53 de l'Orateur du Peuple.

32. Sur ce point, voir aussi Émilie BRÉMOND-POULLE, La dénonciation chez Marat (1789-1791), Révolution Française.net Éditions, 2006, p. 152-154.

33. Archives de la Préfecture de Police (dorénavant APP), AA, carton 32 ; AN, F/7, carton 4625, f. 135 .

34. L'ami de la Révolution ou Philippiques, dédiées aux représentants de la Nation, aux gardes nationale et à tous les Français, $\mathrm{n}^{\circ}$ 19, 15 janvier 1791. Cette « contre-attaque » de Rotondo est conservée en AN, F/7, carton 4625, f. 196 .

35. Sur la protection accordée par les Cordeliers à Rotondo, voir Albert MATHIEZ, Le Club des Cordeliers pendant la crise de Varennes et le massacre du Champ de Mars, t. 1, Paris, Champion, 1910, p. 16-35. D'autres informations intéressantes à ce propos sont dans Alphonse AULARD, La Société des Jacobins : recueil de documents pour l'histoire du Club des Jacobins de Paris, t. 2, Paris, Jouaust-Noblet- 
Quantin, 1891, p. 211-212 ; Isabelle BoURDIN, Les Sociétés populaires à Paris pendant la Révolution, Paris, Sirey, 1937, p. 65-66.

36. Giambattista ROTONDO, Aux citoyens patriotes des sections de Paris, et de toutes les sociétés des amis de la Constitution française, 1 mai 1791 (BnF, coll. 8-LB39-4898). Rotondo nie ses rapports avec le duc d'Orléans: cette accusation avait été soutenue suite à la parution d'une correspondance secrète entre les deux hommes. On peut considérer cette publication comme un faux, forgé par ses ennemis afin de le discréditer : ce texte, intitulé Lettre de M. Rottondo à M. le Duc d'Orléans, est conservé à la BnF, coll. LB39-1538.

37. AN, F/7, carton 4625, f. 191.

38. APP, AA, carton 215 , f. 457. Sur l'opposition populaire parisienne pendant la journée du 17 juillet 1791, voir David ANDRESS, Massacre at the Champ de Mars. Popular Dissent and Political Culture in the French Revolution, Woodbridge, Boydell, 2000, où à la p. 181 il est fait mention de Rotondo. En donnant la nouvelle de l'arrestation du professeur italien avec d'autres personnes "toutes étrangères", Le Moniteur du 19 juillet transcrit l'arrêté récent sur les "étrangers foudroyés" où l'on annonçait que "les mouvements actuels sont le produit des efforts de quelques factieux » auxquels «se joignent d'abord les étrangers payés pour exciter du trouble en France ». Le Moniteur universel, $\mathrm{n}^{\circ}$ 200, 19 juillet 1791.

39. APP, AA, carton 215, f. 458.

40. Adresse du professeur Rotondo à tous les citoyens français amis de la liberté, BnF, coll. LN27-17889. À propos de son arrestation du 17 juillet, Rotondo écrit : « Enfin, le jour à jamais affreux, le jour exécrable où l'on a souillé l'autel de la liberté du sang du peuple, qui ne devait être répandu que pour elle, ce jour a manqué d'être le dernier de ma vie. Je revenais d'une assemblée où j'avais passé deux heures avec le commandant Santerre et un grand nombre d'excellents citoyens, qui tous ont attesté ce fait. Je suis arrêté sur le Pont-Neuf par un nommé Duru, simple soldat du bataillon de Henri IV, et aujourd'hui adjudant de ce même bataillon, grade qui est vraisemblablement le prix de son lâche assassinat ".

41. Le Moniteur universel, Supplément, $\mathrm{n}^{\circ}$ 36, 27 octobre 1793.

42. Paul RoBIQUET, Le personnel municipal de Paris pendant la Révolution: période constitutionnelle, Paris, Jouaust-Noblet-Quantin, 1890, p. 483.

43. Georges LENôTRE, « Rotondi-Rotondo... », art. cité, p. 144.

44. François UZUREAU, Les agents des septembriseurs à Angers, Angers, Grassin, 1914.

45. Louis-Marie de LA RÉVELLIÈRE-LÉPEAUX, Mémoires de La Revellière-Lépeaux, publiés par son fils, t. 2, Paris, Nourrit, 1895, p. 116.

46. Sur les événements révolutionnaires de Rouen, voir Christine LE BOZEC, «Rouen entre tentation et raison", dans Jean-Clément MARTIN (dir.), La Contre-Révolution en Europe, XVIII ${ }^{e}$-XIX ${ }^{e}$ siècles. Réalités politiques et sociales, résonances culturelles et idéologiques, Rennes, PUR, 2001, p. 25-33, qui, tout en refusant une lecture purement modérée de la ville, souligne la résistance de Rouen aux « tentations extrémistes ».

47. AN, F/7, carton 6155, dossier 1133, pl. 1, 8 .

48. Ibid., p. 7.

49. Archivio di Stato di Torino (dorénavant AST), Materie politiche per rapporto all'interno, carton 4, dossier 3, f. 4.

50. National Archives, Home Office 1/1, rapport daté de Londres, le 29 octobre 1792.

51. Ibid.

52. De ce point de vue, le fait qu'au printemps 1793 (alors qu'il commence à être persécuté à cause de ses propos contre la famille royale anglaise prononcés dans les cafés de Londres), Rotondo préfère se réfugier à Genève plutôt que de revenir en France : dans le revirement «diplomatique » qui est alors en cours et qui amène au décret de "non-ingérence réciproque » du 13 avril (Virginie Martin, «La diplomatie en Révolution... », thèse citée, t. 2, p. 169-178), la 
propagande révolutionnaire devient désormais un interdit et Rotondo perd donc ses principaux soutiens politiques parisiens.

53. Le Moniteur universel, $\mathrm{n}^{\circ} 114,24$ avril 1793.

54. D'ailleurs, même si l'on en croit le témoignage de Rotondo, pendant son séjour à Paris, il aurait été en contact avec des politiciens (comme Danton ou comme les girondins Condorcet, Lebrun et Vergniaud) dont les fortunes n'ont pas été liées à l'an II.

55. AN, F/7, carton 6155, dossier 1133, pl. 7.

56. Il décrit ce déménagement comme le résultat d'une opération orchestrée par le Royaume de Sardaigne avec l'aide du gouvernement suisse. Selon lui, les hommes qui l'escortaient avaient pour but de « reconquérir la Savoie et de se rendre tout de suite par cette rue à aider les Lyonnais qui s'étaient révoltés contre la Convention nationale ». AN, F/7, carton 6155, dossier 1133, pl. 7.

57. Le texte de la lettre est dans Le Moniteur universel, $\mathrm{n}^{\circ}$ 262, 19 septembre 1793.

58. AST, Materie politiche per rapporto all'interno, carton 4, dossier 3, ff. 8, 47. Pour plus d'informations sur l'affaire Rotondo-Lamballe, voir aussi Giuseppe ROBERTI, « Un septembriseur italien ", art. cité, p. 65-76.

59. Sur les réseaux contre-révolutionnaires de l'époque à l'échelle européenne, voir Jean-Clément MARTIN (dir.), La Contre-Révolution en Europe, op. cit.

60. Des notes de lectures et des courts passages personnels sont en AN, F/7, carton 6155, dossier 1133, pl. 4 .

61. Cette définition est proposée par le même Rotondo dans une lettre adressée le 21 pluviôse an V (9 février 1797) au chargé d'affaires français à Turin François Jacob: AN, F/7, carton 6155, dossier 1133, pl. 1.

62. Sur le passage de Rotondo à Lucques et sur la politique de Porro visant à un élargissement de la République Cisalpine, voir Antonino DE FRANCESCO, « Genova e l'Italia : il complotto democratico nella pratica politica del Triennio", dans ID., Rivoluzione e costituzioni. Saggi sul democratismo politico nell'Italia napoleonica (1796-1821), Naples, Esi, 1996, p. 38-39.

63. «Benché tutto abbia impiegato e tutto messo in opera per sorprendere la vigilanza del tiranno napoletano e della degna sua sposa (la messalina austriaca), tutto fu inutile, essendovi più spie attualmente a Napoli che forse in tutta l'Europa ». Rotondo continue ainsi sa description de son aventure napolitaine et du climat de suspicion qui règne dans la ville : « En saisissant en deux classes d'hommes les habitants de cette ville immense, je peux avec sûreté vous assurer qu'ils sont pour une moitié des espions vendus à ce bourreau infernal couronné et pour l'autre moitié des militaires ayant les armes à la main le jour et la nuit » [ "Considerando in due classi gli uomini che abitano quella immensa città, con fondamento posso assicurarvi che la metà sono tutte spie vendute a quell'infernale carnefice coronato e l'altra metà è tutto militare sopra le armi giorno e notte »]. AN, F/7, carton 6155, dossier 1133, pl. 6 .

64. Ibid.

65. À ce propos, voir Antonino DE FRANCESCO, « Les patriotes italiens devant le modèle directorial français ", dans Pierre SERNA (dir.), Républiques sœurs. Le Directoire et la Révolution atlantique, Rennes, PUR, 2009, p. 267-280.

66. AN, F/7, carton 6155, dossier 1133, pl. 1.

67. Ibid.

68. Ibid., pl. 9.

69. Ibid., pl. 1.

70. Pour protester contre la réforme de la Constitution de Trouvé, qui est mise en œuvre le 31 août, deux membres importants du parti démocrate de la péninsule (le commandant en chef de l'Armée d'Italie Guillaume Brune et le patriote italien Giuseppe Lahoz) arrivent à Paris au même moment. Si le premier s'y rend muni d'une charge officielle auprès du gouvernement français, la mission de Lahoz n'est en revanche pas très claire: sur cette affaire et, plus 
généralement, sur le profil de Lahoz, nous renvoyons à Francesco Mario AGNOLI, Il giacobino pentito : vita, morte e battaglie del generale Giuseppe La Hoz, Rimini, Il cerchio, 2009.

71. AN, F/7, carton 6155, dossier 1133, pl. 8.

72. Le passage de la lettre de Monti dit: «Je viens de voir grâce à Celentani une lettre de Visconti, où il nous prévient de l'arrestation d'un certain Rotondo, autrefois employé par la police cisalpine. Il a été conduit au Temple. Parmi ses papiers, l'on a trouvé nombre de documents cisalpins, et l'on dit qu'il y en a certains qui témoignent de ses relations avec Testi. Si cela est vrai, mes espoirs de la continuation de la charge de Testi sont terminés. Je n'arrive pas à t'exprimer combien ces nouvelles me rendent triste. Je t'ai perdu, maintenant je perds Testi et peut-être Lamberti, et il ne me reste plus aucun ami dans le Directoire ». ["In questo momento mi si mostra da Celentani una lettera di Visconti, nella quale avvisa l'arresto seguito in Parigi d'un certo Rotondo altre volte impiegato in affari della Polizia Cisalpina. Egli è stato tradotto al Tempio. Fra le sue carte se ne sono trovate molte Cisalpine, e vuolsi che alcune accusino una qualche relazione con Testi. Se questo è vero, le mie speranze sulla permanenza di Testi al suo posto sono finite. Non ti so dire quanto mi rattristano queste nuove. Ho perduto te, perdo Testi e forse Lamberti, e non mi resta più un amico nel Direttorio »]. Alfonso BERTOLDI (dir.), Epistolario di Vincenzo Monti, t. 2, Florence, Le Monnier, 1928, p. 108-110.

73. Archives diplomatiques du ministère des Affaires étrangères, Correspondance politique, Milanais, carton 56, f. 410 .

74. AN, F/7, carton 6155, dossier 1133, pl. 8 .

75. AN, AF/IV, carton 1671, f. 362.

76. « Rome est le centre des intrigants anglais. [...] Les nommés Calenda, Napolitain, et Rotondo, de Monza, se sont réunis, dans cette ville, à un émissaire de la reine de Naples appelé Vincent Mora. De là partent tous les bruits qui se répandent dans les ci-devant légations et ailleurs. Le Citoyen Cacault a dû demander l'arrestation des deux premiers de ces mauvais sujets ». AMAE, Correspondance politique, Milanais, carton 60, f. 280, Extrait des dépêches du vice-président. Des informations concordantes sont données par Francesco Melzi d'Eril, vice-président de la République italienne à Milan, à Ferdinando Marescalchi, ministre des Affaires étrangères de cette République siégeant à Paris : Carlo ZAGHI (dir.), I carteggi di Francesco Melzi D’Eril duca di Lodi, vol. 4, Milan, 1960, p. 138-140.

77. AN, F/7, carton 3777, Bulletin de la police générale, 10 octobre 1812.

78. Jeanne-Louise-Henriette CAMPAN, Mémoires sur la vie privée de Marie-Antoinette, t. 2, Paris, Baudouin, 1823, p. 120.

\section{RÉSUMÉS}

L'analyse de la biographie de l'Italien Giambattista Rotondo, un professeur de langues ayant participé à plusieurs révolutions dans toute l'Europe avant de rentrer à Milan en 1797, permet de réfléchir aux conséquences de la présence des étrangers révolutionnaires tant dans les pays d'accueil que dans leur patrie. Ce cas incite à aborder la question du retour, afin d'enquêter non seulement sur l'action concrète de ces hommes dans d'autres contextes nationaux, mais aussi sur le rôle qu'ils jouent, grâce aux expériences vécues ailleurs, dans leurs pays d'origine. Le parcours de Rotondo s'avère exemplaire à cet égard : si, dans un premier moment, sa participation aux événements du début de la Révolution française lui permet d'obtenir des charges dans la 
République cisalpine, par la suite, la radicalité dont il fait preuve lors de son séjour en France se reproduit en Italie, provoquant l'interruption brutale de sa carrière.

The analysis of the biography of Giambattista Rotondo, an Italian language teacher who took part in several revolutions throughout Europe before returning to Milan in 1797, allows reflecting on the consequences that the presence of some revolutionary foreigners has both in their homeland and other countries. We try to investigate the question of return in order to analyse not only the action of these men in other national contexts, but also the role that they played in their homeland. Rotondo's career is very interesting in this respect. At first, his participation in the events of the beginning of the French Revolution enabled him to obtain some official position in the Cisalpine Republic. Later, the radicalism having characterised his stay in France would be repeated in Italy and interrupts his career abruptly.

\section{INDEX}

Mots-clés : Circulations révolutionnaires, Italie, Triennio républicain (1796-1799), Jean-Paul Marat, Club des Cordeliers

Keywords : Revolutionary circulations, Italy, Republican Triennio (1796-1799), Jean-Paul Marat, Club des Cordeliers

\section{AUTEUR}

\section{PAOLO CONTE}

Université de Basilicate 